\title{
Digital Biomimetic Architecture between Art and Dynamic Structure: Case Study_Wings in Flight
}

\author{
Shi-Yen Wu $\mathbb{D},{ }^{1,2}$ Felicia Wagiri $\mathbb{D}^{1},{ }^{1}$ Yen-Fen Huang, ${ }^{3}$ and Shen-Guan Shih $^{1}$ \\ ${ }^{1}$ Department of Architecture, National Taiwan University of Science and Technology, Taipei 10607, Taiwan \\ ${ }^{2}$ Department of Architecture, National United University, Miaoli City 360, Taiwan \\ ${ }^{3}$ Bartlett School of Architecture, University College London, London WC1E 6BT, UK
}

Correspondence should be addressed to Shi-Yen Wu; sywu@mail.ntust.edu.tw

Received 2 September 2019; Revised 23 February 2020; Accepted 4 April 2020; Published 22 June 2020

Academic Editor: Dehua Shen

Copyright (C) 2020 Shi-Yen Wu et al. This is an open access article distributed under the Creative Commons Attribution License, which permits unrestricted use, distribution, and reproduction in any medium, provided the original work is properly cited.

\begin{abstract}
Architecture as a multirelated field is influenced and connected by many subjects. Among those subjects, the role of art and natural science is the most dominant frame without ignoring the development of advanced technology. By using technology, the impossible becomes possible such as to capture the body motion of humans as the subject of art and science at the same time. Body motion is a potential research of movement over the time. This does not only involve the aesthetic, but also even more the scientific aspect of a dynamic motion of an organism that can be investigated through a biomimetic approach. In order to understand the biomimetic term, we investigated the physical morphogenesis and geometrical principle of an organism. The term morphogenesis is a process in which the natural system produces and regulates the configuration of a material in space and over time. Based on that, we tried to design a dynamic structure using butterfly's motion as a subject of study with morphological and biomimetic approaches. Butterflies show a simultaneous aspect of movement over time characterized by fragmentation. This idea also summarizes many aspects of modern art such as portrayal of body movements by futurists, space-time continuum, cinematic freeze frame, and time-lapse photography. A futurist represents a movement that is emphasized on the factors of speed, technology, modernisms, and objects. This indicates an alternative position that may be relevant to the system of butterfly wings. This can only be achieved by utilizing a digital design and its parametric tools that help generate functions and form dynamic structures with high complexity and precision. Throughout the development of the system, there will be many changes to the form which will be constantly tested and evaluated using a series of prototype and visual digital design.
\end{abstract}

\section{Introduction}

Over the last 50 years, design and architecture have grown rapidly (and will continue to grow) to different phases and tried to break down their defined boundaries and started to integrate with other disciplines. Amongst many disciplines, visual arts and natural sciences such as physics, mathematics, chemistry, and social sciences, have constantly affected architectural thinking. The crossover results between these disciplines have resulted in a new form of collaboration and a new way of understanding the architectural context to promote a fresh perspective on design and architecture through the creative art processes and computer science that demonstrate reciprocal relationships.
The implementation of collaborative work was successful in generating a multidisciplinary attitude as well as achieving a new awareness of current technological advances. In this case, collaborative works discuss the potential partnerships between architects and artists in the creative interplay of Futurism and Dynamism. Futurism is an Impressionism of the mechanical world that uses advanced technology and urban modernity. It is committed to the new things, destroying older forms of culture and showing the beauty of modern life, machine, speed, and change [1]. The sensibility of the futurist is characterized by everything that is alive, which always responds and offers everything that moves, and whatever exists in motion (Dynamism and simultaneity). 
There are many attractive works, ranging from Futurism and dynamics to creative applications in painting and art projects. The movement of the human body expresses mobility of space and sensibility of time and analyzes the potential for movement over time, involving many artistic activities such as dancing, theater, and spatial performing arts in both biological and psychological substances. The idea of motion is focused on the flux of memories and the sensations of what we remember, as well as the physical movement of what we see. The movement includes not only physical changes but also emotional and social changes. The artists are offered the possibility of motion visualization that not only featured the aesthetic aspect but also even more the scientific aspect of dynamic motion of body movement. The combination of physical movement of body, space, and time has been explored in another discipline that is chronophotography [2]. Dynamic motion can be seen from the artwork of cubists, where a story is formed by the movement of images with a painting. The overall transformations and positions are highly dependent on the physical motion of the body when one or more variables are changed within a given time frame.

Dynamic motions can also be found from morphological and biomimetical approaches with the goal of implementing into various dynamic structures in response to diverse backgrounds such as art, science, technology, and architecture. With a wide range of newly available tools, interdisciplinary collaboration makes it possible to investigate biomimetics. In order to understand the term of biomimetics, we can investigate the physical and geometrical principle of morphogenesis. Morphogenesis is known as the process by which the natural system produces and regulates the configuration of matter in space over time [3]. This movement is a great organizational pattern in a dynamic system. The same components can be configured differently to produce endless transformable structures depending on their positions and interconnections in space, and these can operate in two or three dimensions that form many possible shapes. Movement in this context refers to the physical motion of the organism. This refers to perceptions formed by spatial sequences, optical illusions, and composition changes that bring a feeling of movement, which may be experienced differently by each observer. On the other hand, time as a design element greatly enriched the possibilities in the development of this dynamic structure (see Figure 1). Structures can be designed by following coordinated movements and action of time, acting similarly to living organisms. In this case, movable elements can act on the rhythm, sequence, periodical, cyclical, repetitive, and diverse progressions. Furthermore, the dynamic structure involves consideration of abstract and qualitative issues concerning the awareness of technology, spatial experiences, and social engagements.

\section{Technology in Science and Art}

Technology and science sometimes "do art" and art "does science and technology" [2]. The borders between art and science have completely disappeared altogether. Technology and industrial products are always implemented into art creations that will always use the best newly available technology. Industrial products always use the best newly available technology to do art creations even in the past and yet still do today. Not only technology is being increasingly used, but the technology also becomes the creative art itself.

By using computer technology, it becomes possible to analyze the diversity and simulate the processes that occur in nature, structures from behavioral patterns, complex shapes that change over time, and habits that affect the environment and its physical form. Technology enables one to manipulate data through computer systems and machines that then make it into a three-dimensional object that has the potential to mimic the complex forms of nature that traditional construction cannot touch (see Figure 1). With the machine, it can grow a unique 3D shape that represents a new order in design evolution.

This dynamic field of computer technology tends to cooperate with art and science (based on Figure 1), trying to explore each new visualization possibilities with a new approach to the art process. Nowadays, computer is not only a tool, but also a cocreator because even though the artist has set and started certain algorithms, she/he cannot predict the end result of computer activity according to the algorithm. This technical invention has a major influence on art techniques and the creative process. Without it, there will be no graphic or photographic or digital media technique that opens up further possibilities of the related art discipline. In the dynamic technology, there are some basic approaches to visualize motion art, static (photography and images), or dynamic (movies and animations). Artists and dancers use cameras to catch their performance, whereby they have specific forms and types of photographs, which range start from artistic dance photography to the performance documentation and the study of movement.

The transformation of photography is an example of big invasion in technical applications of art which is naturally becoming an achievement of science and industry. Discipline like this can also be seen from the application of new media art, 3D printing, and computer visualization. However, examples of science and art can be found further in history that has discovered remarkable mathematical visualizations [4]. The principles of mathematics, geometry, and arithmetic are introduced not only to science and technology but also in art. When looking at the produced works throughout history, beauty is often associated with perfection of mathematical order, as Da Vinci frequently did. This order has been widely explored in nature, and artists seek to study it in detail to transfer it into painting, sculpture, and even architecture.

Neal Panchuk in "An Exploration into Biomimicry and its Application in Digital and Parametric Architectural Design" presented to the University of Waterloo, Canada, in 2006, said that by the rise of Postimpressionism, an improvement occurred, which in the development of science and technology embarked on a new paradigm of "being an imitation" to copy nature. Biomimicry is an applied science that derives inspiration from the study of nature. There is a relationship of nature with genetic coding. It is not to form a genetical code of an organism but rather the process of selfgeneration within the environment. Geometry has a subtle 


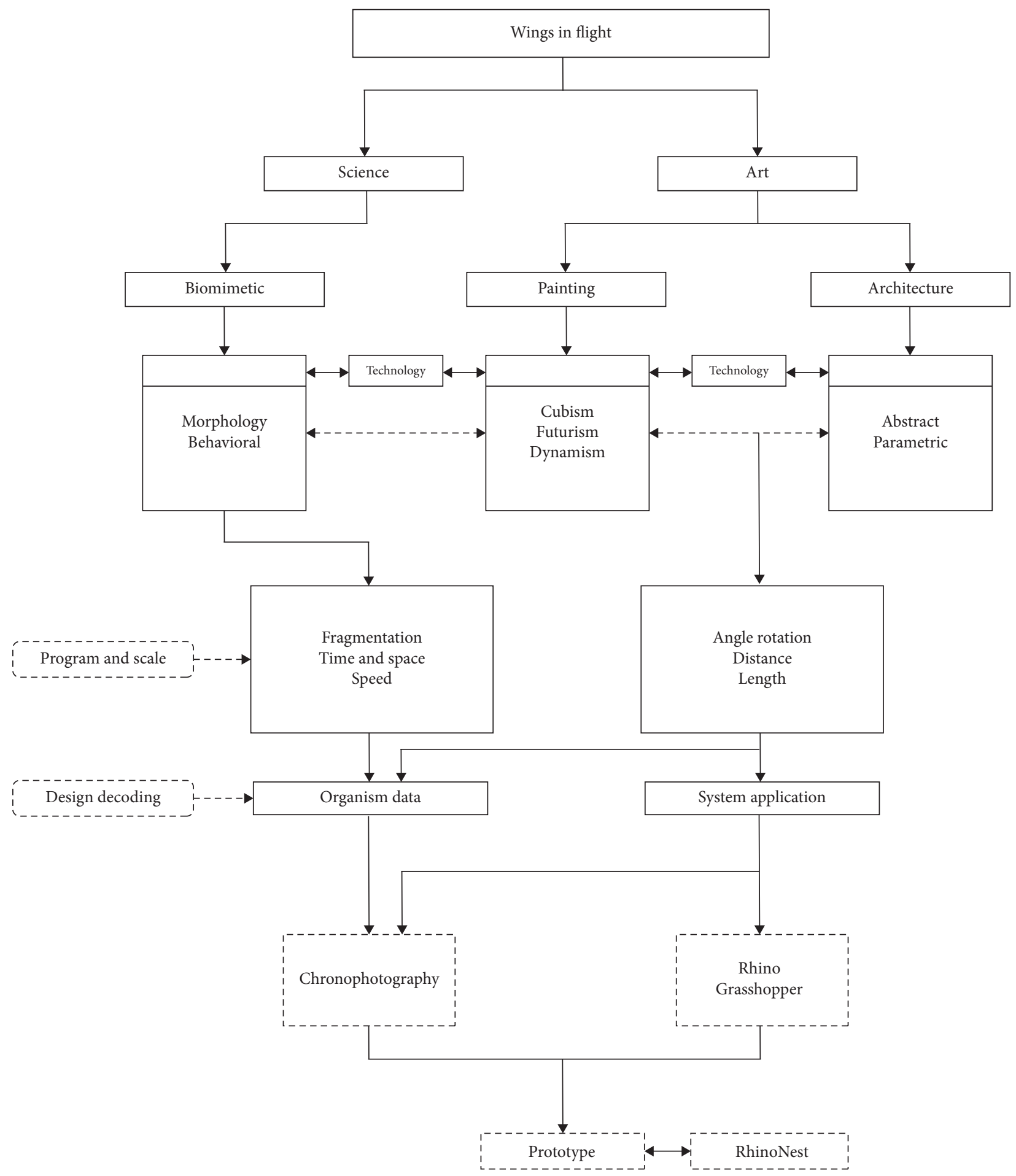

FIGURE 1: Diagram relationship between science and art (source: drawing by the research team).

role in morphogenesis [5]. It is necessary to think about biological geometry or computational forms not only as a description that is fully developed but also as a principle of local organization in self-organization during the process of morphogenesis.
2.1. Learning from Nature. In the book "The Pulse Concept and the Study of Biomimicry" published by the SCAD School of Design in 2014 cited Baumaister who said that biomimicry is the conscious emulation of nature's genius. It is an interdisciplinary approach that brings together two often 
disconnected worlds, nature and technology, biology and innovation, and life and design [6]. This biomimicry seeks to bring the living wisdom of time tested and life practice into a design to inform humans in creating conditions that are conducive to sustainable living. In practical biomimicry, how to find sustainable solutions is to examine life's blueprints, chemical prescriptions, and ecosystem strategies. However, at the level of transformative biomimicry, it connects us with the most appropriate, aligns, and integrated way to the human species into the natural process of the Earth.

Biomimicry approach is seen as a design process that can be divided into two categories, namely, defining the human needs or design problems and seeing other organisms or ecosystems work as a solution to solve the problem. The socalled solution is design look for particular biology to identify its characteristics, its behavior, and its function in an ecosystem which is then translated into a human design that may affect biology [7].

When architecture is considered, there are many obvious distinctions between biomorphism and biomimicry at any level. Biomorphic design or architecture is rooted in the work of surrealists and Art Noveau. A surrealist, Grefory Grigson, coined the term "biomorphism" in 1936. Since then, the term of biomorphism has been combined with computation to replicate the visible free-form of nature because by looking at nature, we can create edges, surfaces, and volumes [8]. It is also possible to use this knowledge to find a form that resembles a living entity, but its function is not adapted. In this design approach, the results stay only at the visual level, not referring to functional and ecological levels.

Through the study of existing biomimetic technology that is still developing today, it is clear to see that there are three levels of mimicry, they are the organism, behavior, and ecosystem. The first level of biomimicry is to mimic the natural form of an organism. This type of mimicking is to copy the morphological attributes of an organism such as the visual form, component, material, or appearance. Design at this level can look fancy, but ecological and functional perspectives are not necessary at this level [8]. Morphology has been transformed from naturalism to schematic stylization in which an animal is formed abstractly, geometrically, and almost unidentified. Biomimicry at a deeper level, the second level, is mimicking the natural process. Imitating the natural process means imitating the behavioral pattern of an organism. This can be achieved by exploring and understanding how an organism relates among species within its own environment. It is possible to imitate relationships between organisms or how they behave. This requires a deeper understanding to make ethical decisions about the compatibility of what can be copied to the human context. This level is not suitable for all situations and contexts at all times. The third level is to imitate the natural ecosystem. This is a complex series of processes than the first and second levels. At this stage, the mimicking ecosystem should consider the designed objects and how those objects affect the environment explicitly and implicitly [9]. By extending the sphere of influence, the correct sustainable approach can be applied. In addition, the results are functional, sustainable, and also beautiful. Beauty is the most important part why biomimicry at any level resonates.

2.2. Art and Futurism. In the book "History of Graphic Design, A Reference to Important Events," it was mentioned that the future includes speed, technology, youth, and violence. Futurism influences many movements such as Constructivism, Surrealism, Art Deco, and Dadaism [1]. Futurism is practiced almost in every medium of art, such as sculpture, painting, ceramics, theater, film, fashion, graphic design, interior design, and architecture. Futurism is the Impressionism of the mechanical world. It uses the most advanced technology and considers urban modernity, refers to a new style, destroys the older forms of culture, and demonstrates the beauty of modern life-machine, speed, and changes. Unlike many other modern art movements such as Impressionism and Pointillism, Futurism is not easily recognized and understood because it is distinctive and influenced by Cubism.

The sensibility of the futurist is characterized by a passion for what is alive, which always moves and offers whatever in motion (Dynamism and simultaneity), in contrast to whatever in static. It is also dedicated to designs with high complexity which can only be achieved by machines and computers. Futurism requires interpretation precisely. The beauty of speed is the key element of Futurism. Futurists were influenced by new developments in visual technology, more specifically chronophotography, predecessors of animation and cinema that allow the movement of objects to be shown on a sequence of frames. Technology has an important influence on their approach to show movement, encouraging the abstract visual art with rhythm and pulsation.

Dynamism of a Dog on a Leash by Giacomo Balla in Figure 2 was fascinated by chronophotography, a technique in which movement changes are shown on several frames. This concept encouraged Balla to explore and discover new ideas to represent movement and rhythm in painting, and Dynamism became one of his most famous painting experiments.

Figure 2 shows a woman walking with a small black dog in which the movement melts into a single instant. It is showing a close-up of the feet. Balla articulated the action in a process by incorporating opaque and semitransparent shapes. This painting is a rapid-fire exposure that expressed the true dynamics of humans and animals in action and motion.

Chronophotography pioneer Eadweard Muybridge (1830-1904), an eccentric photographer, is famous all over the world for successfully photographing animal and human movements imperceptible to the human eye (see Figure 3). He was studying motion using several cameras from many angles once in a time, capturing essences and sensations of motion and speed. In 1872, Muybridge used photography to prove that there was a moment in horse's gallop, a time when all four hooves were off the ground at once (see Figure 3). In 1878, Muybridge has successfully photographed a horse in 


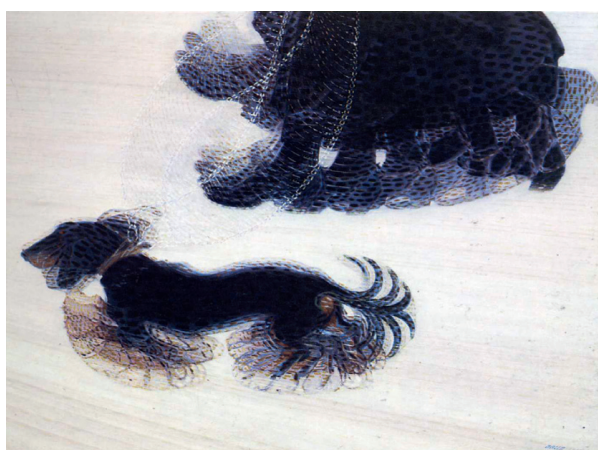

Figure 2: Dynamism of a Dog on a Leash by Giacomo Balla using chronophotography [10].

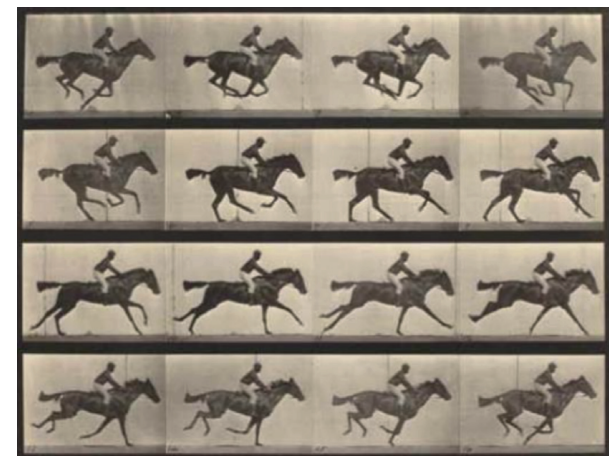

Figure 3: The Horse in Motion (1872) by Eadweard Muybridge [4].

fast motion using a series of twenty four cameras. Muybridge used 12 stereoscopic cameras, 21 inches apart to cover the 20 feet taken by a single horse step, taking pictures in a thousand seconds.

The other great mind in the late 19th century was Etienne-Jules Marey. He is a scientist, a psychologist, and also at the same time a chronophotographist. His vision in motion and time is used to measure a beating heart and to capture birds in flight, resulting in technology leading to modern cinema. In Figure 4, he used such brilliant ideas to capture and record "The Man Walking" into several phases of movement in one photographic surface [4].

Some of the cubist artists, futurists, or readymade artists, e.g., Marcel Duchamp, were involved in various modern art movements and were responsible for excellent examples of futurist paintings that implied motion and time, titled Nude Descending the Staircase (1912) as shown in Figure 5. As can be observed, Duchamp offered his own interpretation of Muybridge's Woman Descending the Staircase. By using the abstract form of a naked woman who could be identified as she was moving down the stairs in the painting [4].

Motion is made much more explicit in nude paintings. Duchamp developed and refined the swirling lines and staccato arcs from points that describe the progress and displacement of the moving subject [11]. There is a relationship between Nude Descending a Staircase and Futurism. A futurist concerns on movement. By using popular chronophotography at the time, he was able to capture the movement of an organism over a period of time. The nude painting also leads us to Cubism in decomposing forms [12].

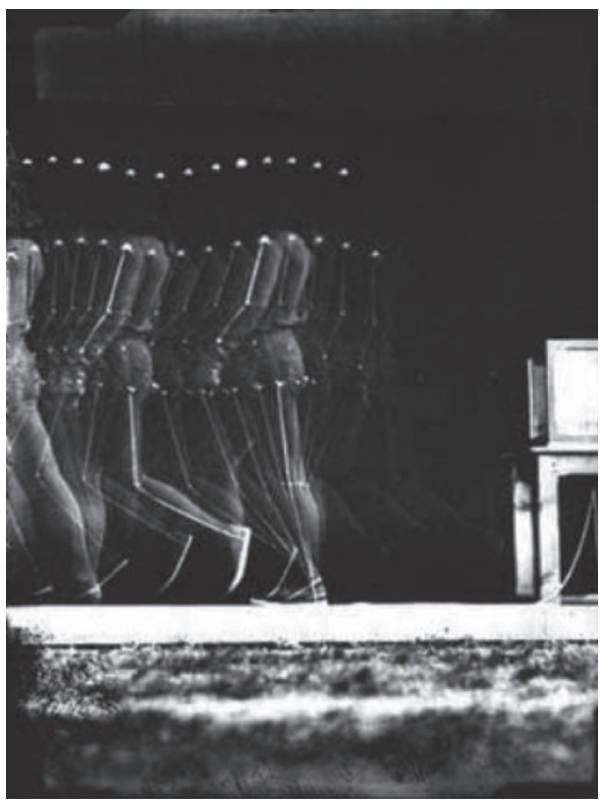

FIgURE 4: The man walking (1890) by Etienne-Jules Marey [4].
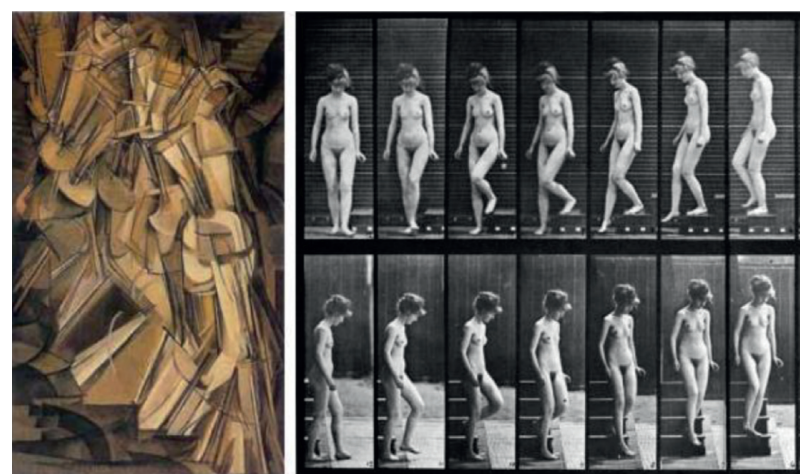

Figure 5: Nude descending the staircase (1912) by Marcel Duchamp; woman descending the staircase (1891) by Eadweard Muybridge [4].

\section{When Science and Art Becomes One}

In the modern era, science is aimed at applications, technical advancements, and mastery of nature. Art has no such purpose, its scope is symbolization, transmission of cultural meanings, human development, and self-interpretation in specific historical environments. To achieve these goals, art uses all the available advances that exist with exceptional ingenuity, including findings in various areas of expertise, scientific discoveries, and the invention of technological applications [4].

The examples listed above show that the production of contemporary art not only changes the boundaries between art and science but also redraws the line between the viewer and the creator. In many cases, viewers play an important role in the work, but in other cases, they are even able to inform the final form itself. The similarity is found in scientific and technological progress which does not only affect every aspect of our everyday life but also becomes a new art media that is often used in the process of art creation. 
In every dynamic cultural development, art and science is known as a twin engine of creativity. Science tries to understand natural phenomena by using scientific methods including observation, experimentation, and testing, as well as formulating hypotheses. The field of science is focused not only on science itself but also extends to the realm of social and culture. Art, on the other hand, especially in the past, is characterized by the application of a respected time-honored media such as painting, graphic arts, and sculpture. They are created for the purpose of aesthetic experience. With the emergence of new artistic means and daring experiments with the latest technological advances, the boundaries between art and science have gradually disappeared. In the book with the title "Art + Science," Stephen Wilson refers to an institutional art theory with which he thinks the definition of art is dynamic and shaped by whatever network of art and artists, curators, historians, and critics consider to be accepted [2].

Although there are many theories that define the differences between the two entities, Stephen Wilson provides arguments based on the fact that the cave painters have become intensive investigators in the field of zoology, anatomy, and psychology. Their paintings reveal a sophisticated understanding of animal life processes. He further argues that by revealing the history of science or art, we will find prehistoric cave paintings the first important milestone in both. He points out that Leonardo Da Vinci and several others have the idea of "deep seeing," which means understanding the basic processes of the world (kind of what scientists do), and is seen as an essential tool for making art. For example, studying the flow dynamics can help an artist to paint water, studying flight mechanics can help artists while painting birds, and investigating anatomy and dissection allows the artists to become a better painter or a body sculptor [4].

3.1. Digital Biomimetic Architecture. In the last 20th century, architecture became the first art which is gradually used by designers who work with digital technology in the scope of arts. Architecture is also influenced by nature and living organisms. This includes a focused understanding not only on designing a better environment for humanity in the future in order to preserve ecological diversity, but also related to the concept of "Futurism" that provides interesting information and ideas in the creation of space and latest innovative designs with high complexity using computation technology. The focus of this concept will not only become the answer to sustainability, but more than that, it will also explore the process of a sustainable future and mobilize people to think of sustainability by mimicking the morphology of an organism [13].

Architectural morphology was introduced by Philip Steadman in his book entitled "Architectural Morphology: An Introduction to the Geometry of Building Plan." It focuses on geometric limitation primarily related to its boundaries, structure, and design. He often uses the phrase "morphology" which refers to the general science of potential forms such as natural forms, art forms, and architectural forms.
The emergence of the morphogenetic concept in its development shows the value of algorithms in pursuit of nonstandard and organic forms. Computation technology is considered as a new method of form-finding. One of the advantages of form-finding is the possibility of combining multidisciplinary research such as computational geometry and biomimetic patterns [13]. Conversely, in computational logic, the threshold depends on the Boolean value. The effectiveness of the algorithm calls for principle consideration. We must give the computer clear rules and orders to generate a form corresponding to the design ideology, in which it may be mathematical, biomimetical, or performative.

An author Leonardo in 2005 published a biologybased procedure to generate an experimental digital architecture. The text originated from Louis Sullivan's morphological design process articulated in the Architectural Ornament System. He said that the biomimetic architecture combines architectural paradigms with digital calculations, generative scripts, advanced fabrication, biomaterials, and nature that play an important role in the development of new systems, shapes, structures, aesthetics, and materials. This approach can integrate a procedure for understanding, visualizing, producing, and forming the architecture by studying the process in nature. For example, looking at seeds, diatoms, algae, shellfish, etc., can be a suitable biomimetic information to the possibility of new architectures. This new architecture considers how sustainable environments can become integral to new technical innovations and attitudes toward genetics, biomaterials, and science collaborations. The algorithm will also consider how the forms can follow some natural methods, such as those found in butterflies. Thus, biological growth determines body, behavior, and movement, resulting in a structure or a mechanical system that grows digitally for architecture.

Technology is one of the most important driving forces in the development of modern architecture. With technology focused on the digital computation design, it can provide flexibility in designs where it is possible to change the shape through parameters without having to change and redesign the entire form. Parametric design can be updated automatically through parameter changes. These systems are related to each other to inform the overall form. No one knows what will be the final outcomes because the result is unimaginable when using a computation program known as generation. By mimicking an organism, algorithmic and parametric designs show that the architecture can borrow from nature and generate the possibilities of infinite forms, defying previously set boundaries of knowledge. In the design parametric, everything can be manipulated from initial analysis and design process to the final form, materiality, fabrication, and construction, and control points are given to allow for a degree of flexibility [14]. The parametric design not only creates a generation of responsive forms for buildings, but also creates a space that can be adapted for future use. Architects now face a digital period that shifts from composition to generation. 
3.2. Art and Dynamic Structure. Art is a kind of creativity that is communicated through visual, verbal, spatial, and audible aids, whether in the form of real phenomena or subjectively subjected experiences, abstract ideas, emotions (both in intellectual and aesthetic), and body sensations. To create a work of art, artists need at least a creative skill or competence in a particular field, but most artists in generative art leave some creative process to be done by a machine. By using computer technology, it is possible to analyze diversity as well as to simulate processes occurring in nature, ways of behavior, and complex shapes that can change over time (dynamic).

There are many exciting art works, ranging from various technology that uses human body motion as a subject through video simulation to creative applications in painting. The movement of the human body is a subject of research that has the potential to analyze movements over time as it involves many artistic activities such as dancing, theater, and performing arts that are inherently spatial in both their biological substance and psychological abilities. The artists are offered more possibilities of motion visualization, which not only display the aesthetic aspect, but also even more the scientific aspects of the dynamic movement. Digital motion tracking offers a broad field of investigation in Dynamism, continuing into ecological psychology, perception, memory studies, creativity studies, media studies, biomechanics, and the history of ideas.

In all ages, performers such as dancers and actors always use their bodies as the main elements of their creations and make their motion artwork recorded. In contrast to painters and sculptors, they use a physical form in their work to capture a "real-time" experience that cannot be repeated and only happens once; therefore, it is rare and unique in time. Petra Sobanova in his book titled "Useful Symbiosis: Science, Technology, Art, and Education" explained that the capture of elusive events with a particular media depends on the technical possibilities of a certain era of humans (drawings, paintings, chronological photography, cinematography, and video) [4]. This capture shows the dynamic movement as a set of fixed points in a movement continuum that explain the idea in every movement which can be captured graphically through different graphical representation techniques including drawing, 3D modeling, diagrams, and notation.

The movement developed in a dynamic motion as a new form of design derives interest in exploring its variables, testing its limits, and benefiting from its potential. This paper tries to design a dynamic movement of morphological and biomimetical approaches using butterflies as a study case with the goal of implementing it to various dynamic structures in response to a unique set of inputs from diverse backgrounds such as art, painting, science, technology, and architecture. Throughout the system development, it will continue to be tested and evaluated from a series of physical and digital prototypes.

While studying the morphology of dynamic structures, further elements have to be considered which are those related to the changes that may occur during the movement process. The morphological analysis takes a synergistic view in which a composite system of components is considered to achieve the overall transformation of its parts. Obvious variations are needed to be made between art and the dynamic structure to create motion and time that exist in space. This creates a dynamic space and space of movement, which represents the forces of life and produces a form of structure or architecture. Basically, architectural theories and practices have involved mobility in terms of physical movement of the inhabitants, the sensation of motion as a result of the visual effects of changes in lighting or even the presence of moisture, the existence of humidity, and the representation of movements with forms and structures that show Dynamism of the futurist art.

The futurist idea represents movement in a picture which struck the cubists, in which the painter seeks to capture coexistence of separate ideas or mental experience in an instant. Duchamp painted a picture that moves the world with the concept of Cubism. In a book entitled "Cubism" written by Guillaume Apollinaire and Dorothea Eimert, five movements of the movement of one person, descending a spiral staircase and captured on time lapse were mentioned [15]. For Marcel Duchamp, the goal of the personal style is fragmentation. Movement of fragmentation of this movement cultivates futuristic aesthetic. Fragmentation is a form of resistance to force destruction, to preserve the most important part of the whole and to reconfigure a difference in the future. Fragmentation often occurs during crisis times.

\section{Case Study}

The design is focused on biomimicry at the morphological and behavioral levels, where form and natural process will be studied further. The design is inspired by butterflies and related to the possible (interdisciplinary) future, where collaboration flows naturally in terms of architecture, biology, and technology. Milad Arkian in his research about butterfly, entitled "Temperature Control Mechanism by Butterfly Wings," stated that butterflies are known to live and survive under delicate environmental conditions with extreme weather changes. They are biologically cold blooded, and some form of basking is needed to raise their body temperature. They use their wings effectively to regulate body temperature. They require a heat-control mechanism to prevent overheating due to wing flapping, but body temperature must be high enough to be able to do a relief flight [16]. The purpose of this design is to investigate the biomimetic processes of the butterfly while regulating and maintaining their body temperature by modeling and understanding the principle pattern of wings' movement.

4.1. The Uniqueness of Butterfly's Aerial Feats. Birds and insects fluttering for a balanced flight in the air show taking off and landing vertically, alternately, and drift. A butterfly is a right model for describing the type of vehicle with an autonomous microaerial system because of the frequency of packing itself.

A research about the flying mechanism of a butterfly was done by three Japanese researchers, Masahiro Shindo, Taro 
Fujikawa, and Koki Kikuchi, and successfully published by the international journal and publication named ATEAS (American Transactions on Engineering and Applied Sciences) in 2014. In that study, the position of the butterfly body is shown as in Figure 6 with symbols to illustrate the angle of roll, pitch, and yaw. Horizontal and vertical body planes symbolized by $\mathrm{ZB}$ and $\mathrm{XB}$ are defined as normal regulators. The angle rolled, depicted, and yaw are symbolized as follows: $\varphi \theta \psi \cdot \varphi$ is the angle between the horizontal body plane and the $Y$ axis, $\theta$ is the angle between the horizontal body plane and the $X$ axis, and $\psi$ is the angle between the vertical plane of the body and the $Y$ axis [17].

\subsection{Behavioral Thermoregulation. The flight parameters} describing the state of the butterfly are selected as follows: the angle between the horizontal body plane and the front wing in a normal vector is defined as the flapping angle. The flapping cycle is divided into two phases, namely, the upper and lower strokes [17].

The three main postures shown in Figure 7 are typical butterfly postures for thermoregulation, and they are lateral, dorsal, and reflectance. Lateral basking is a state when wings are closed over the body and oriented perpendicular to the sun's rays. At the dorsal basking, the butterfly opens its normal wings into the sun rays so that it instantly heats the wings, thorax, and abdomen [16].

Photos in Figure 8 show behavioral illustrations when a butterfly is flapping its wings taken by a high-speed 3D camera system [17]. These pictures are designed to show how the butterfly's frame corresponds to loops and lines.

Butterflies body represent flying or flapping simultaneously in a terrible rush. The multiplication and motion effects allow forms to expand this movement. Figure 8(a) shows the streamlines around the wing caused by the flapping motion. The streamline color corresponds to the flow velocity, where the red line represents faster and the blue line represents slower [17]. The wake capture mechanism explains the increase in aerodynamic force during stroke reversal. Figure $8(\mathrm{~b})$ shows the constant pressure surface on the wing as a stroboscopic image. The red and blue surfaces show positive and negative pressures. Positive pressure is on the upper side and negative underneath during the down stroke.

4.3. Implementation. Butterfly is an aspect of a simultaneous movement which is characterized by fragmentation and manipulation of the structure, which serves to distort and dislocate some elements. The design represents the aerodynamic characteristics during the roll rotation. This makes it possible to analyze flow fields that match the behavior when the butterfly is flying. Overlapping figures are used to increase the impact of flying motion to the viewer. Everything moves, everything runs, and everything changes rapidly. The figure is never stationary but appears and disappears constantly. Through the persistence of images on the retina, things in movement multiply and distort, succeeding each other like vibrations in the space through which they pass.
The structure is formed by the image of the butterfly motion exploring the techniques of mimicking, imitating, capturing, analyzing, deconstructing, and reconstructing the movement of butterflies in a dynamic spatial perspective. The entire structure is simply based only on repeating elements consisting of various slices of wings' movement and then represented together to imply their relationship to each other. The distance of each unit is different and floats separately. This can be seen as a time lapse from a higher interval to a lower interval. This design uses several different static positions creating a sense of motion and visual movement that a futurist has claimed. The idea of motion is focused on the flux of memories and the sensations of what we remember, as well as the physical body movements of what we see [18]. The movement does not only embrace the physical changes but also the emotional changes.

It has been concluded that the dynamic system which is involved in the production of forms differs fundamentally in biological and systematical. First, the dynamic form is the result of complex movements across scales and angles. Second, the model depends on the unit interacting in the dynamic way where everything depends on time lapse and interval. The connection between units and the overall shape is fundamental in relation to the dynamic system of the movement of a butterfly (Figure 9).

The movement of a butterfly is a great organizational pattern in dynamic systems. The same components can be configured differently to create transformable structures that are endless. Depending on their position in space and interconnection, they can operate in two or three dimensions that make up many possible shapes. Movements in this context indicate the physical motion of an organism. This refers to the perception of space formed by spatial sequences, optical illusion, and compositional changes that can bring a feeling of movement, which may be experienced differently by the observer [19]. Fly-free displays the importance of the time dimension in relation to space. Either it is invariant from space-time or a complete representation of objects. The time-space interval is constant because the length and time of the transformation are inversely related. The smaller the length of the unit, the greater the time. As a time interval increases, the length decreases, and as a length increases, the time interval decreases.

On the other hand, the introduction of time as a design element greatly enriches the possibilities in this dynamic structure. Structures can be designed to follow every script where movement and time are coordinated, acting similarly to living organisms. For this instance, moving elements can operate following rhythm, sequence, or progression, and movement can be periodical, cyclic, repetitive, and diverse. Furthermore, introducing dynamic systems into the design will involve consideration of abstract and qualitative issues concerning on awareness, spatial experience, social engagement, and user interactions [19].

Dynamic structure involves a setting of variable ranges and angles to change over a period of time. Variables must be modifiable and identified as length, angle, and position. The transformation and the overall position depend on the physical movement of the butterfly when one or more 

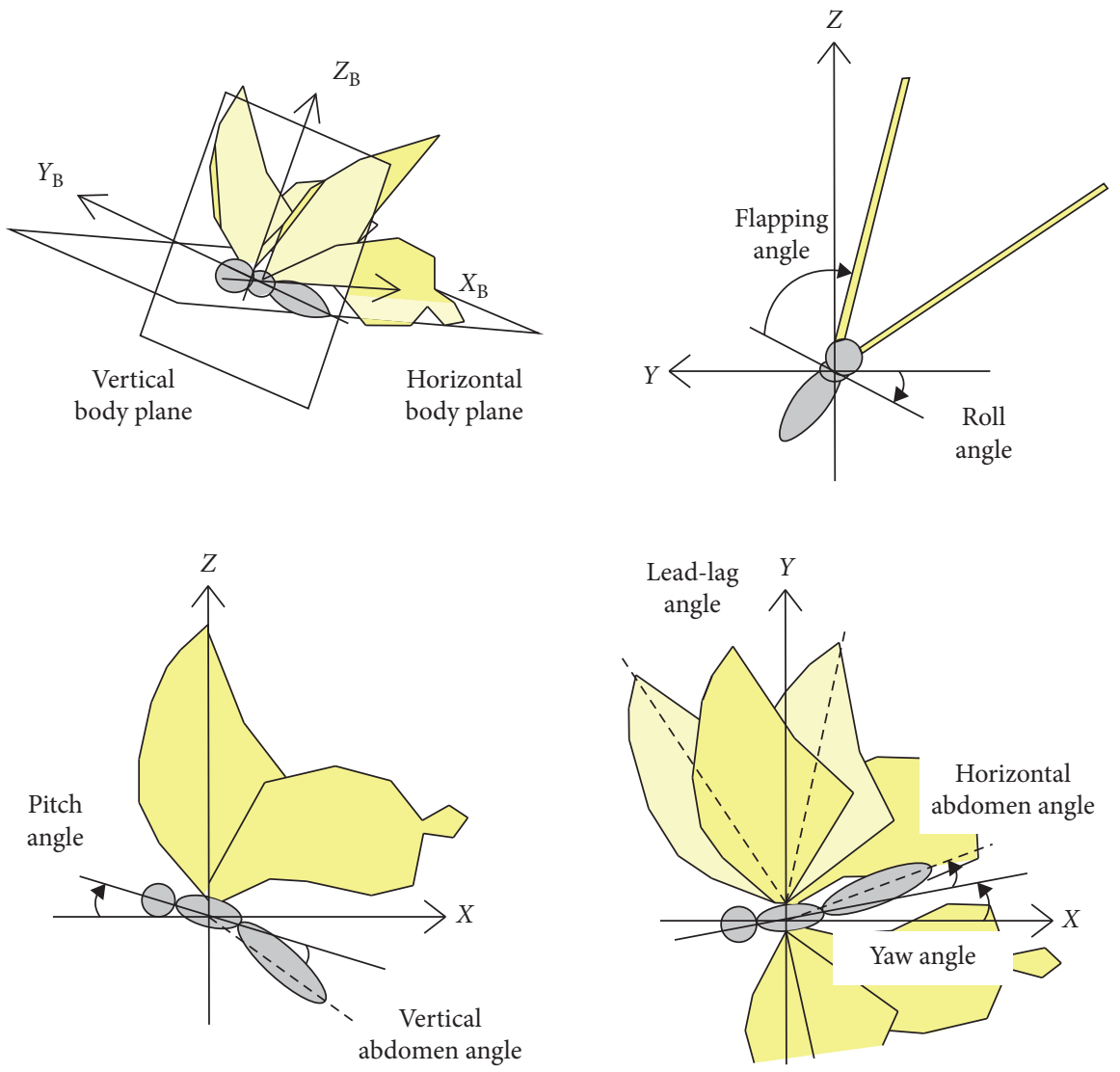

Figure 6: Definition of the butterfly frame, angles, and posture, picture from [17].<smiles>C=c1ccc(=C)cc1</smiles><smiles>C=c1ccccc1=C</smiles><smiles>C=c1ccc(=C)cc1</smiles>

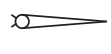

$$
\theta=0^{\circ}
$$$$
\gamma=90^{\circ} \text { or } 270^{\circ}
$$

Lateral

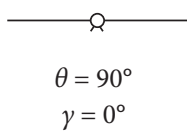

Dorsal

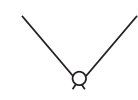

$5^{\circ}<\theta<90^{\circ}$ $\gamma=0^{\circ}$

Reflectance
FIGURE 7: Basking postures: lateral, dorsal, and reflectance used by butterflies [16].

variables are changed within a specific time frame. These variables can be defined as different points that later define the form of the curve. Each curve serves in a different plane with different Graph Mapper as the controller (see Figure 10).

The fourth dimension is built from the similar geometry that we normally visualize [18]. As explained in Figure 10, it starts with a zero-dimensional point, and then by moving that point in any direction for any length creates a line (and an $x$-axis). Moving the line perpendicular to the $x$-axis creates a plane (and an $x$ - and $y$-axis). Then, moving the plane perpendicular to both the $x$-and $y$-axis creates a space (an $x$-, $y$-, and $z$-axis).
This is the logical system that is used to easily understand the image of objects in a three-dimensional space system, regardless of how distorted it is because of its intuitive to the space perspective. As shown in Figure 11, by using the analogy of transformation, moving space perpendicular to the $x-, y$-, and $z$-axis creates the fourth dimension. However, being trapped in three-dimensional space, we cannot visualize the fourth dimension coordinate system, or what objects are in the fourth dimension. Two main methods for representing the fourth-dimensional objects, slicing methods and projection methods, have been developed as an attempt to make the invisible visible [18]. For a more complex design, not only as a sine curve, it is necessary to tweak the points in both planes, $x$ and $y$ points in the XY plane and $x$ and $z$ points in the XZ plane (see Figure 11).

Minkowski referred to his four-dimensional space-time formulation about the theory of relativity, "space by itself and time by itself are doomed to fade away into mere shadows, and only a kind of unity of the two will preserve an independent reality" [18]. Since three-dimensional geometry only relates to space, it cannot explain the implications of relativity. For a four-dimensional graph, that is what Minkowski and Einstein believe in our universe, in the field of the Cartesian plane, $z$-axis and $y$-axis must be released, and it is easier to see the two variables that are subject to change, $x$ and $t$. Since time always flows through our universe and the contraction of length only affects $x$-axis, $z$-axis and $y$-axis can be taken out of consideration. 


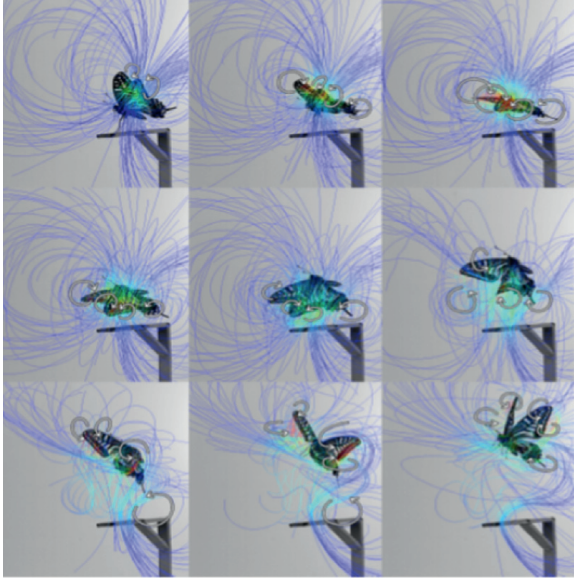

(a)

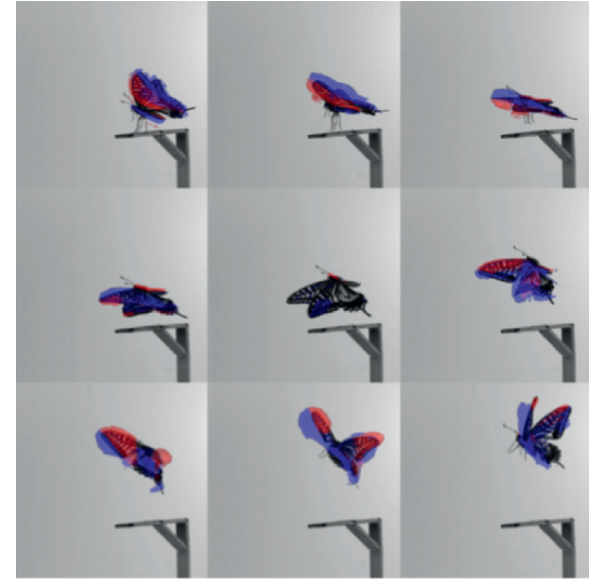

(b)

FIGURE 8: Stroboscopic image pitch rotational flight picture from [17].

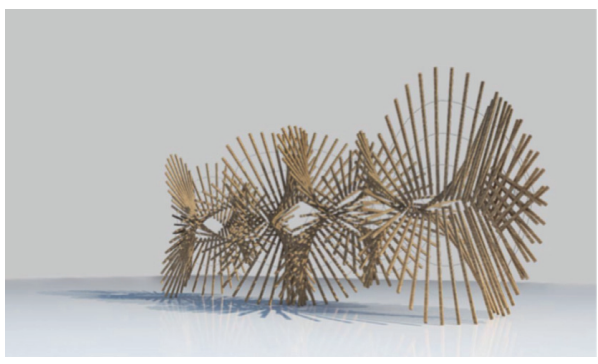

(a)

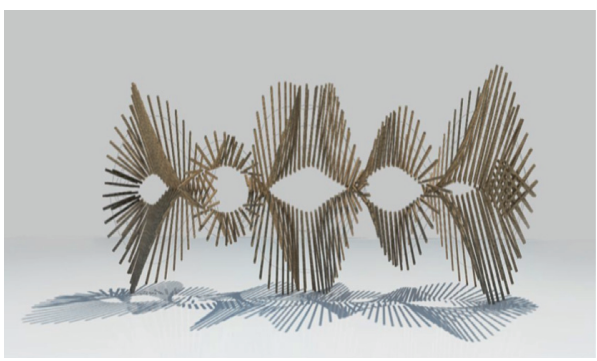

(b)

FIGURE 9: Implementation structure of butterfly flying movement. Concept: wings in flight (source: drawing by the research team).

Default values for graph mapper 0-1

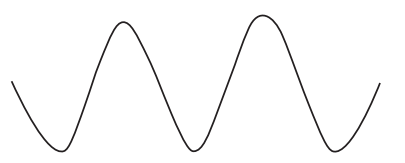

0

Graph mapper for y's $\quad 1$

Desired values for graph mapper 0-8 (The range is adjustable)

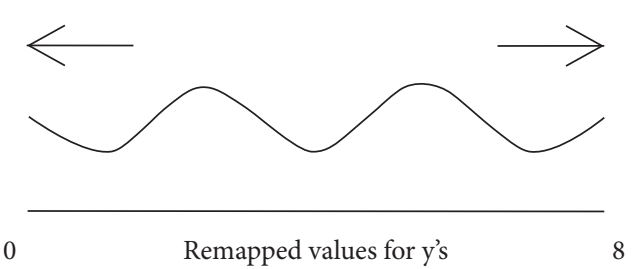

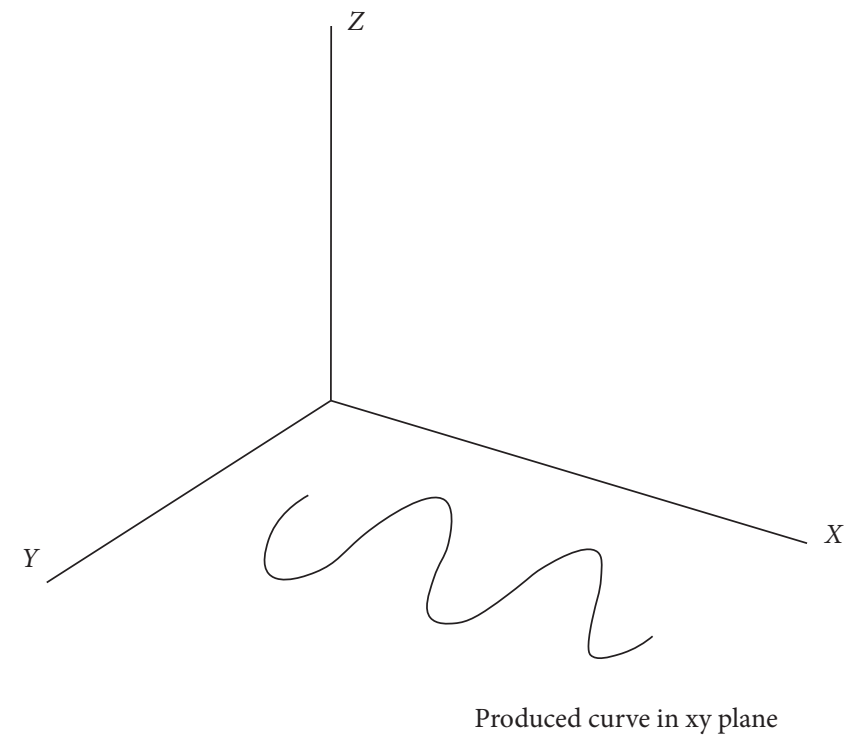

(a)

Figure 10: Continued. 
Default values for graph mapper 0-1

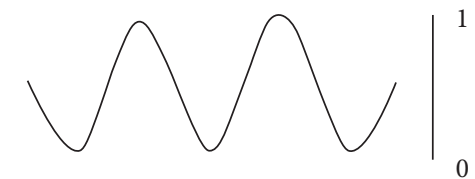

$0 \quad$ Graph mapper for z's $\quad 1$

Desired values for graph mapper $0-4$ (The range is adjustable)

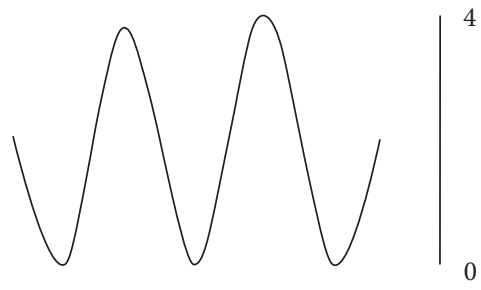

Remapped values for z's

FIGURE 10: Logic static system of the model, inspired by the free movement angles (source: drawing by the research team).

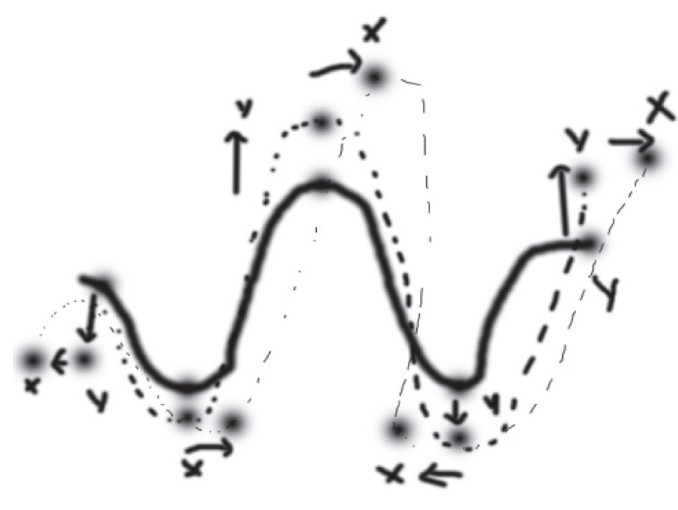

(a)

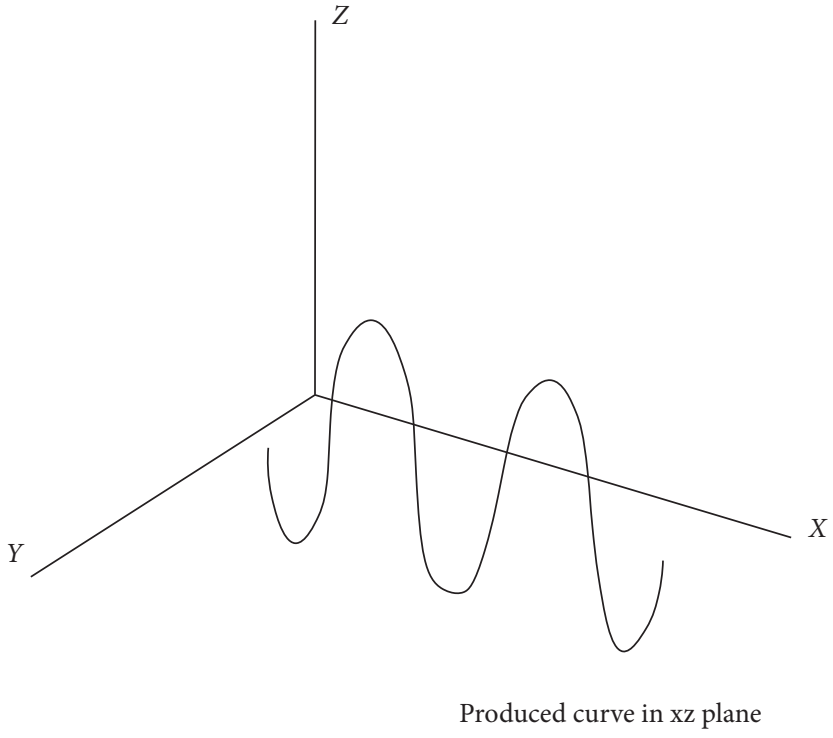

(b)

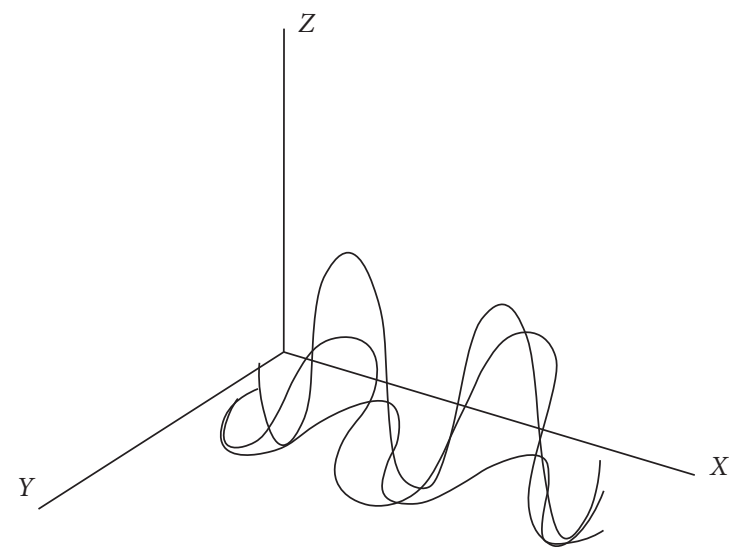

Combination of Ys and Zs

FIGURE 11: Curve on the combination of $y$ and $z$ planes (source: drawing by the research team).

Using that geometry principle, a graph of something would look like a vertical line, as time passes and the object length is constant at a single speed. The faster the something moves through space, the steeper the slope of the graph becomes. In Minkowski's mind, because space and time can both change shape or dilation or contraction, respectively, the only way to understand the true nature of an event is to incorporate two variable things into invariable space-time intervals.

The space-time interval is constant because according to Lorentz, the transformation between length and time is inversely related. The smaller the length of something, the larger the time. As the time interval increases, the length decreases, and as the length increases, the time interval will decrease, but the time interval remains the same since the ratio between the two is an inverse relation [18].

4.4. Fabrication from the Digital Data to the Real Object. To test the fabrication and assembly, prototype structures were built on a smaller scale $1: 10$ using $4 \times 4 \mathrm{~mm}$. Figure 12 shows the prototype image from various views. The model 


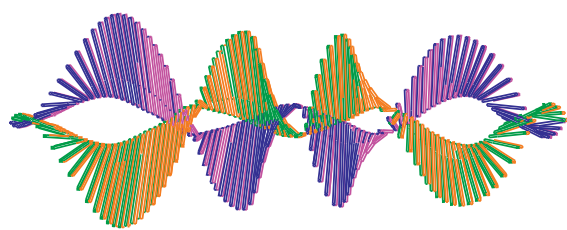

(a)

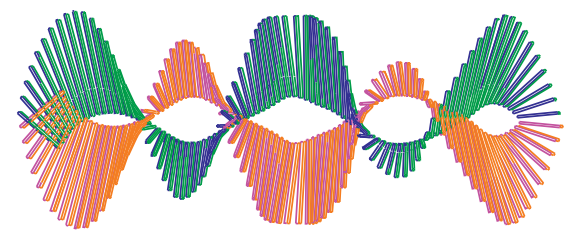

(c)

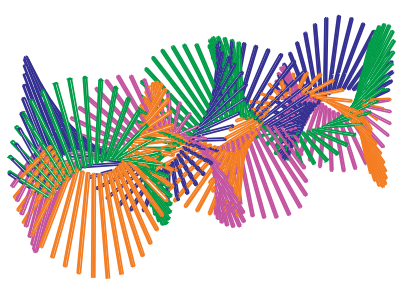

(b)

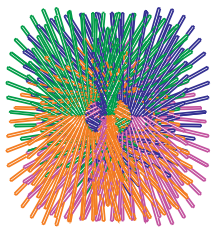

(d)

Figure 12: Case study of complex parametric model using the grasshopper logic system (source: drawing by the research team). (a) Top view_1:100. (b) Perspective view_1:100. (c) Front view_1:100. (d) Right view_1:100.
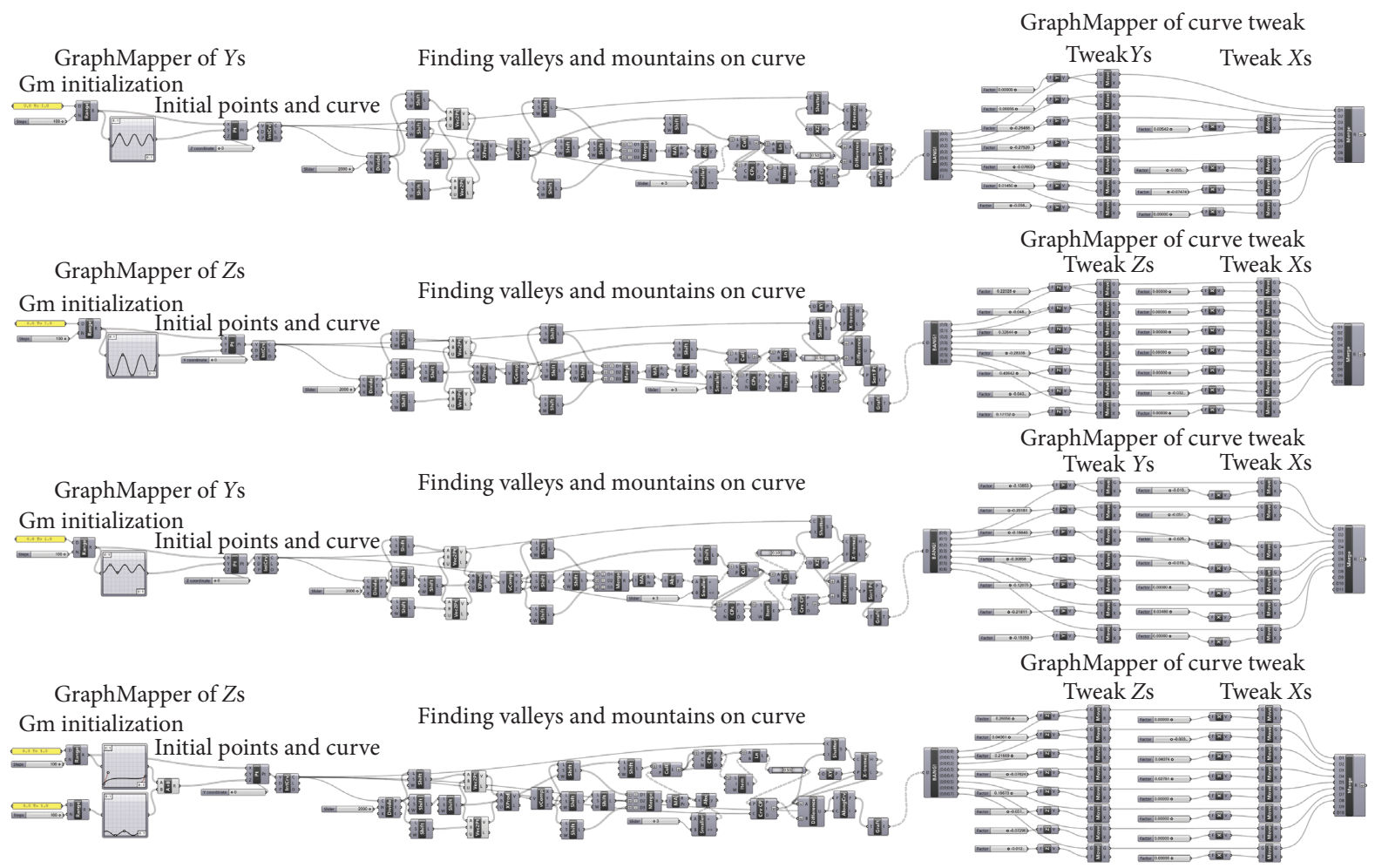

Figure 13: Case study of a complex parametric model using the grasshopper logic system (source: drawing by the research team).

consists of four parts identified by four different colors (blue, magenta, orange, and green). Each part consists of 34 pieces of wood. In total, there are 136 wood pieces connected by wires.

Using a computational genetic algorithm, the design starts with points and connects vertical points and then follows the arc pattern to illustrate the movement of the flying butterfly (see Figure 12). The surface is generated by the cross-linked wood elements with random angles along the structure with the aid of a genetic algorithm, which is then reflected through the XY plane to obtain a complex flight system fluidity. The angle represents the movement of a butterfly that flows in flight to regulate their body temperature.

The main algorithm produces a sine curve that has three mountains and two valleys. The initial points on each plane (either XY plane or XZ plane) are randomly determined by the Graph Mapper in Grasshopper (see Figure 13). In order to get a complex wood structure with cross-linked elements, a set of data for tweaking curves should be defined and merged to get one sine curve. For making other parts, sine curve can be mirrored horizontally and vertically (see Figure 14).

The second step after the outer curves have been determined is to generate the wood pieces. The size of the wood 


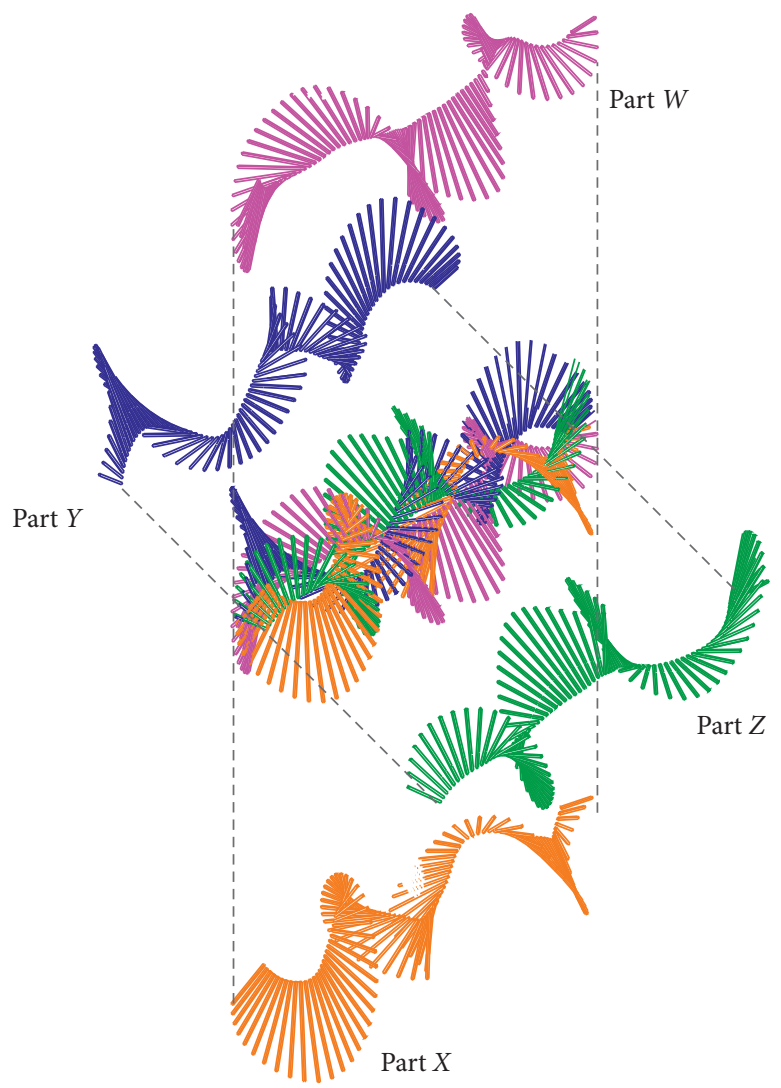

FIGURE 14: Making other parts by mirroring one part horizontally and vertically (source: drawing by the research team).

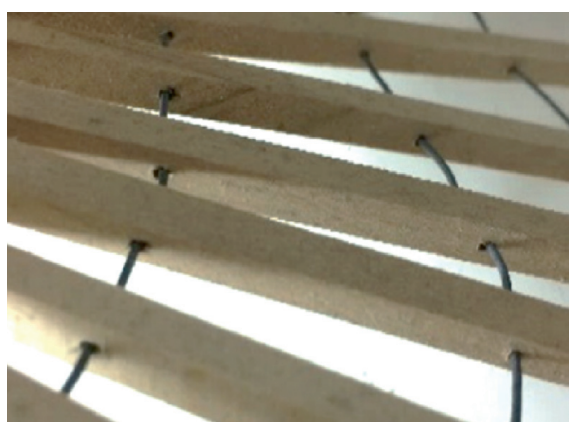

(a)

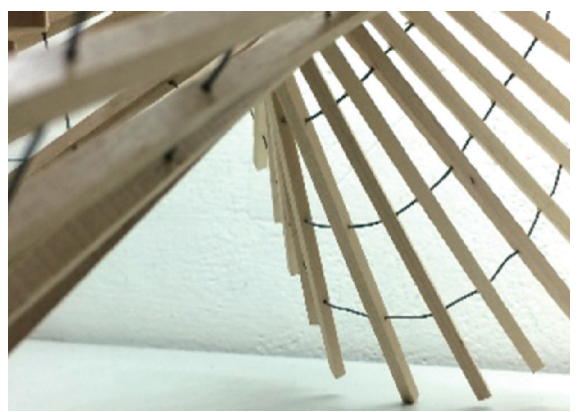

(c)

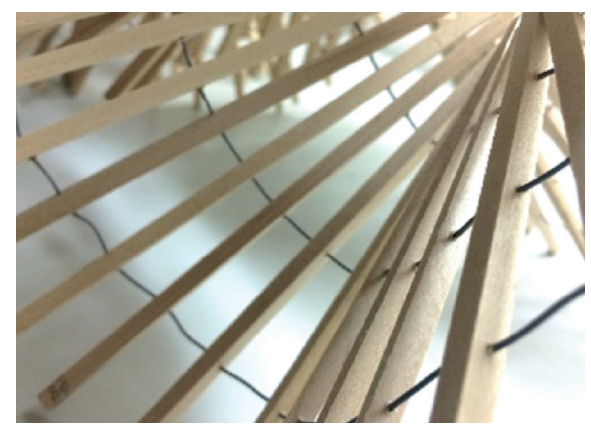

(b)

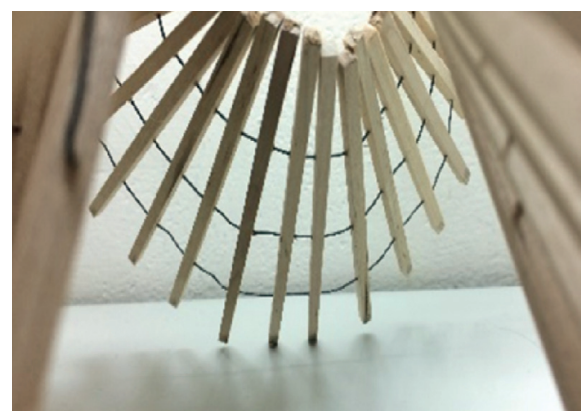

(d)

Figure 15: Wire connection of the complex parametric model (source: drawing by the research team). 
$(4 \times 4 \mathrm{~mm})$ is adjustable, but the length of the wood follows the outer curves that have been generated in the previous algorithm. The total amount of mountains and valleys depends on the total length of the prototype model for a stable structure. After the wood pieces are defined, the hole's marks for the wire connection can be created. In this prototype, three wires with different lengths were used (see Figure 15).

The part is assembled in an oblique position, inserting pieces connected by wires as shown in Figure 15. Between parts, the connection is very difficult because there is no general assembly direction that can be found to determine the distance between woods and the slope angle of each wood. At the full-scale structure, it is easier to use industrial robots for assembly.

Fabrication using industrial robots can produce more precise and accurate results because of their ability to perform an infinite number of nonrepetitive tasks, thus they are considered as the enablers for deeper transformations in the field of large-scale manufacturing systems. For this purpose, further research is needed, using an articulated robot for cost-effective and potential fabrication process. Only in this way, we can significantly expand our range in bio-inspired analysis, architectural design, and production aims, allowing the diversity and complexity of new materials to emerge.

\section{Conclusion}

When looking at architectural biomimetics made through digital design for Futurism, it connects traditional art (movement captured by painting) and new expressive science that allows us to analyze diversity and also simulate the processes that occur in nature through complex patterns of behavior that change from time to time. In order to achieve that, computers and industrial robots play an important role in defining and creating new complex architectural forms and spatial relationships. The relationship between art and science and their overlapping tendencies seems like a thought with postmodern influences and Cubism associated with mobility in terms of physical motion of the occupant, the sensation of motion as a result of visual effects. Representation of movements by forms and structures shows a Dynamism of the futurist art. Art should also be able to reflect the important phenomena of human existence and living organisms, as well as logical problems that science is completely indifferent.

The evolution of architecture from the traditional way to the modern era of computer-based design has really improved in various aspects such as designing, documenting, and idea communication and now stepping forward in integrating scripts, algorithms, and codes to provide a more efficient way of solving complicated problems in the design process, beyond the roots of representation of existing architectural forms in which it is limited by design techniques. In addition, it is important to emphasize that despite a much higher degree of flexibility and speed in generating possible outcomes in the realm of digital architecture, this situation must be considered very well, so that the existence of this technology means capable of serving and bridging the gap between humanity and complexity of the design process.

The design and construction process in this case study is a good reflection of computational methods that helps architects and designers to achieve innovative results and allows more design possibilities. There is always a need for changes in architectural methods when there is a change in human needs and human evolution, but the important thing is to learn the relationship between many fields which are interconnected to each other.

\section{Data Availability}

The data used to support the findings of this study are available from the corresponding author upon request.

\section{Conflicts of Interest}

The authors declare that they have no conflicts of interest.

\section{Acknowledgments}

The research case studies have been conducted in the Department of Architecture, National Taiwan University of Science and Technology, by the Ministry of Education, Taiwan. Additional expenses have been covered by authors' home research centres, namely, the D\&A Lab, Department of Architecture, National United University, and the Department of Architecture, National Taiwan University of Science and Technology, in Taiwan.

\section{References}

[1] P. B. Meggs, A History of Graphic Design, a Reference to Important Events, Wiley, Toronto, Canada, 2016.

[2] S. Wilson, Art +Science Now, How Scientific Research and Technological Innovation are Becoming Key to $21^{\text {st }}$ Century Aesthetics, Thames and Hudson, London, UK, 2010.

[3] P. Steadman, Architectural Morphology an Introduction to the Geometry of Building Plans, Moma Thames and Hudson, London, UK, 1983.

[4] P. Sobanova, Useful Symbiosis: Science, Technology, Art, and Art Education, Palacky University Olomouc, Olomouc, Czechia, 2016.

[5] P. Neal, An Exploration into Biomimicry and its Application in Digital Parametric Architectural Design, University of Waterloo, Ontario, Canada, 2006.

[6] N. Baker, The Pulse Concept and Study of Biomimicry, SCAD School of Design, Savannah, GA, USA, 2014.

[7] S. Virmani, "Architecture and nature: biomimicry as a tool for sustainable architectural design," Vastu Kala Academy, New Delhi, India, 2014.

[8] Y. Arslan, Biomimetic Architecture a New Interdisciplinary Approach to Architecture, Yeni Yuzyil University, Istanbul, Turkey, 2011.

[9] D. Baumeister and R. Tocke, Biomimicry Resource Handbook A Seed Bank of Best Practices, Create Space Independent Publishing Platform, Scotts Valley, CA, USA, 2013.

[10] Albright-Knox Art Gallery, Dinamismo di un cane al Guinzaglio, Albright-Knox Art Gallery, Buffalo, NY, USA, 1912, https://www.albrightknox.org/artworks/196416-dinamismodi-un-cane-al-guinzaglio-dynamism-dog-leash. 
[11] C. Tomkins, The World of Marcel Duchamp, Time Inc., New York City, NY, USA, 1966.

[12] J. Nelson, Wisdom-Conversation with the Elder Wise Men of Our Day, W. Norton \& Co., New York, NY, USA, 1958.

[13] Harper Collins, Innovation Inspired by Nature Work Book, Harper Collins, New York, NY, USA, 2007.

[14] T. Fry, Design Futuring: Sustainability, Ethics, and New Practice, pp. 1-16, Berg, Oxford, UK, 2008.

[15] G. Apollinaire and D. Eimert, Cubism. Art-History-Modern (Late $19^{\text {th }}$ Century to 1945), Parkstone Press, New York, Ny, USA, 1st edition, 1980.

[16] M. Arkian, Temperature control mechanism by butterfly wings, 3rd year mechanical engineering initial report.

[17] M. Shindo, T. Fujikawa, and K. Kikuchi, "Analysis of roll rotation mechanism of a butterfly for development of a small flapping robot," in Proceedings of the 3rd International Conference on Design Engineering and Science (ICDES2014), MM Publications, Pilsen, Czech Republic, August 2014.

[18] E. Bodish, Cubism and the Fourth Dimension, University of Montana, Missoula, MT, USA, 2009.

[19] C. M. Stevenson, "Morphological principal: current kinetic architectural structure," in Proceedings of the International Adaptive Architecture Conference, London, UK, March 2011. 